\title{
THE AESTHETIC APPLICATIONS OF INTENSE PULSED LIGHT USING THE LUMENIS M-22 DEVICE
}

\author{
Yehuda Ullmann ${ }^{1}$, Rania Elkhatib ${ }^{1}$ and Lucian Fodor ${ }^{1,2}$ \\ 1: Department of Plastic Surgery, Rambam Health Care Campus and Faculty of Medicine, \\ Technion-Israel Institute of Technology, Haifa, Israel \\ 2: Department of Plastic Surgery, First Surgical Clinic, Emergency District Hospital, \\ Cluj-Napoca, Romania
}

\begin{abstract}
Background: Fifteen years have passed since the first Intense Pulsed Light (IPL) devices were introduced into the market. A variety of devices that use light energy for aesthetic puposes are currently manufactured by several companies, and newer devices offering improved performance and features are periodically being introduced. Herein we present our experience with the MP-22 device (Lumenis Ltd.,Yokneam, Israel) for cosmetic treatment of benign skin lesions.

Patients and Methods: We collected patient data from all patients who received IPL and Nd:Yag laser treatments with the MP-22 over a one year period (Jan 2009 - Jan 2010). The study group included 92 patients who were treated on the face, neck, trunk and hands. The main indications for treatment were lentigo and superficial vascular lesions. Patient age ranged from 16 to 72 years old, and 89 patients were female. Exclusion criteria for treatment were suspicion of skin cancer, isotretinoin treatment during the previous 6 months, and pregnancy. Satisfaction after treatment was evaluated by the patients on a scale ranging from 0 to 5 , with $5=$ excellent, $4=$ very good, $3=$ good, $2=$ moderate improvement, $1=$ mild improvement and $0=$ no improvement.

Results: Ninety-three percent of the patients were satisfied (rating 3, 4 or 5 on the above scale). Ninety-five percent of the patients had one or two treatment sessions. Of the treatments, 82 were on the face, two on the neck, four on the trunk and 13 on the dorsal aspect of the hands. Minor side effects such as erythema for longer than 24 hours and facial edema for more than one day were reported by 9 patients. Major complications such as scars, hyperpigmentation or hypopigmentation were not recorded. The fluence delivered varied from $14-18 \mathrm{~J} / \mathrm{cm}^{2}$.

Conclusion: IPL is an effective and safe method for skin treatments, namely removal of vascular and pigmented lesions.
\end{abstract}

Key Words: Intense Pulsed Light, benign skin lesions, lentigo

\section{Introduction}

Intense Pulsed Light (IPL) devices employ flash lamps that emit noncoherent light, which distinguishes them from laser devices. It has been over 15 years since the first IPL devices were introduced into the aesthetic

\section{Addressee for Correspondence:}

Yehuda Ullmann, M.D.

Department of Plastic Surgery, Rambam Health Care

Campus

8 Ha'Aliya Street, Haifa 35254, Israel

Tel: +972-525361222 / Fax: +972-4-8542149

E-mail: y_ullmann@rambam.health.gov.il medicine market, and since then, a number of companies have been introducing newer devices offering improved performance and features. The MP-22 model IPL device manufactured by Lumenis Ltd (Yokneam, Israel) features a dual platform containing an IPL head and an Nd:Yag laser. The emitted light is filtered through various filters adjusted according to the lesion and skin type. The light spectrum ranges from $515 \mathrm{~nm}$ to $1200 \mathrm{~nm}$.

The light is absorbed by the chromophores with-

Manuscript received: September 21st, 2010

Accepted for publication: December 3rd, 2010 
in the skin, principally the melanin in the pigmented lesions, and hemoglobin with its derivates (oxy- and deoxyhemoglobin) within the blood vessels. The chromophores absorb different light wavelengths, and the absorbed light is transformed into thermal energy, causing damage to the chromophores and the lesions where they are located. This principle, called: "Selective Thermolysis", was first introduced by Parrish and Anderson ${ }^{1,2)}$ in 1983.

The selective thermal damage of the pigmented lesions within the skin caused by the IPL destroys the lesions, but because the pulses of light are very short, the skin itself remains intact. However, the emitted heat has been shown to cause some positive histological changes, mainly of the collagenous component; synthesis of collagen type I and II is increased ${ }^{3)}$ due to some destruction caused by the heated chromophores. ${ }^{4)}$ An increase in procollagen, elastin and collagenase activity has also been described. ${ }^{5)}$ This new collagen formation is responsible for changes in the skin texture. ${ }^{6)}$ Improvement in skin color gained after the procedure is due to the destruction of melanin and hemoglobin after the IPL treatments. The pigmentary improvement was thoroughly analyzed by Yamashita ${ }^{7)}$ who demonstrated that after IPL treatment, the melanosomes from the epidermal basal layer migrate towards the skin surface.

The collagen changes caused by the procedure are claimed to improve not only the skin color, but also the skin texture, with an end point of total skin rejuvenation. Herein we present our experience using the MP-22 device for skin rejuvenation.

\section{Patients and Method}

We collected data from all patients who underwent IPL treatment using the MP-22 over a one year period (Jan 2009 - Jan 2010). The study group included 92 patients who underwent IPL procedures on the face, neck, trunk and hands. The main indications for treatment were lentigo and superficial vascular lesions. Patient age in the study group ranged from 16 to 72 years old, and 89 patients were female. Exclusion criteria for the treatment were: suspicion of skin cancer, isotretinoin treatment during the previous 6 months, and pregnancy.

Treatment protocol All patients were given a detailed explanation of the procedure and possible side effects and complications, and all gave their informed consent. Most of the patients received their first treatment at the first visit. EMLA cream was used as a local anesthetic in patients for whom large areas were to be treated. Small areas were treated without any analgesia, and for vascular lesions, mainly telangiectasias, we avoided using local anaesthetic creams in order to prevent vasoconstriction that can impede treatment results. No IV sedation was used. The amount of time that EMLA was in contact with the skin varied from 30 minutes for facial lesions to 60 minutes on the hands. The cream was covered by a plastic transparent sheath in order to prevent evaporation. Before treatment, the analgesic cream was wiped off and the skin was cleaned with a wet gauze. A transparent gel was applied on the area to be treated. For the first treatment, the device manufacturer's suggested, pre-set parameters were used according to lesion and skin type; if a subsequent treatment was indicated, the paramaters were adjusted accordingly. After treatment, the area was cooled with local wet gauze for about 5 minutes. No restrictions regarding using makeup or facial creams were indicated to the patient. The patients were scheduled for a follow-up visit within 6 weeks, at which time a decision regarding the need for subsequent treatment was made, with the adjusted parameters.

\section{Results}

Most of the treated patients had Fitzpatrick skin type II (46\%) and III (52\%), with only 2\% having type IV (Fig. 1). The main treated areas were: face ( $83 \%)$, the dorsal aspect of the hand (24\%), and the trunk (5\%) (Fig. 2). The indications for the treatment were: hyperpigmentation (46\%), telangiectasias (32\%), and general facial rejuvenation $(22 \%)$. The number of sessions performed

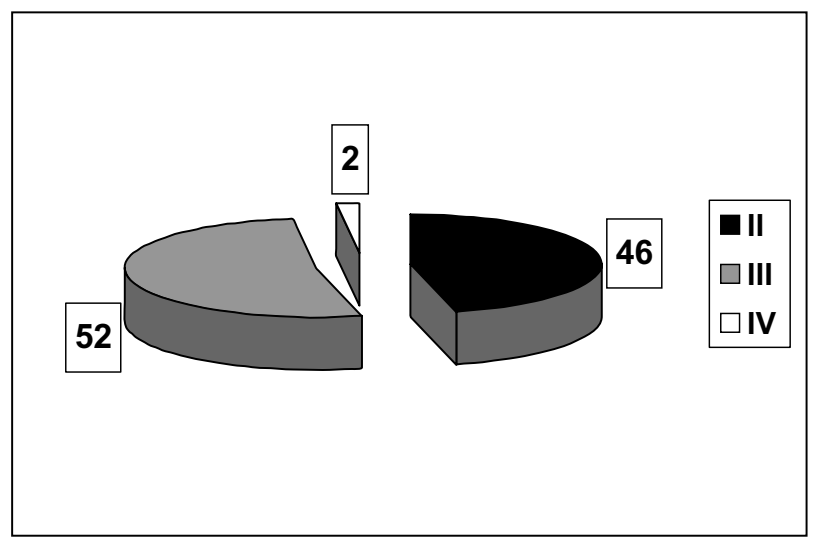

Fig. 1: Skin types treated according to Fitzpatrick classification 


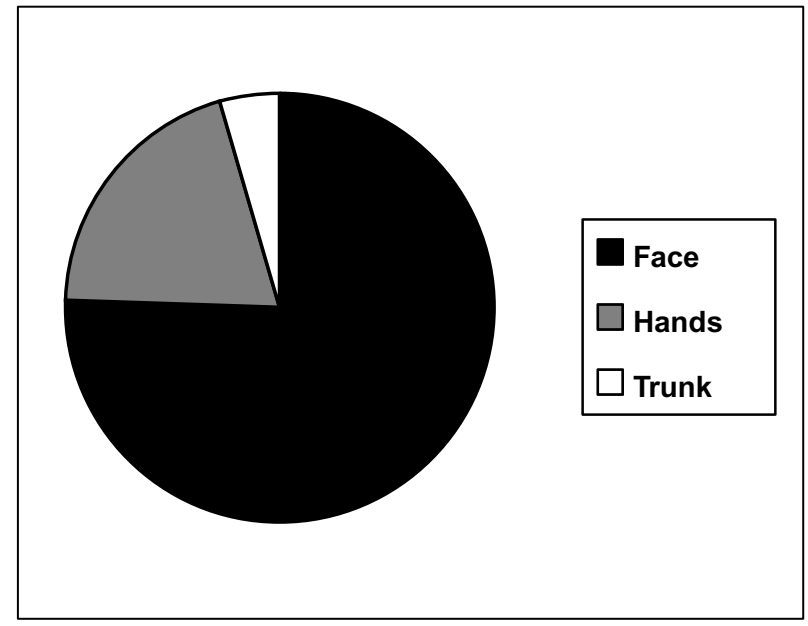

Fig. 2: The Treated Areas

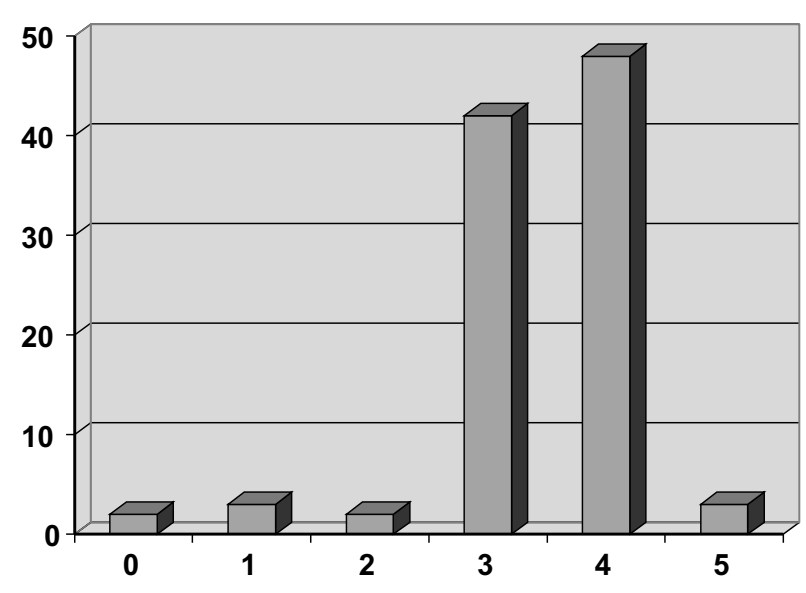

$5=$ excellent, $4=$ very good, $3=$ good, $2=$ moderate improvement $1=$ mild improvement and $0=$ no improvement.

Fig. 3: Patient satisfaction (\%) for each patient were: 1 (60\%), $2(35 \%), 3(3 \%)$, and 4 (2\%).

Satisfaction after treatment was evaluated by the patient on a scale from 0 to 5 , with $5=$ excellent, $4=$ very good, $3=$ good, $2=$ moderate improvement, $1=$ mild improvement and $0=$ no improvement. A nurse from the Plastic Surgery Department collected the data from the patients concerning satisfaction levels. Ninetythree percent of the patients reported being satisfied (3, 4 or 5 on the above scale, Fig. 3).

Minor side effects such as erythema for longer than 24 hours and facial edema for more than one day were reported by 9 patients. Major complications such as scars, hyperpigmentation or hypopigmentation were not recorded. The fluence delivered varied from $12-$ $18 \mathrm{~J} / \mathrm{cm}^{2}$.

\section{Discussion}

The M22 device is composed of a universal IPL system and a Nd:YAG module. An additional feature of the M22 is its optimal pulse technology, which enables the emission of multiple-sequential pulses that allow tissue cooling between each pulse, thereby reducing the chance of skin damage.

The main indications for IPL treatment are removal of pigmented lesions, vascular lesions, hair, and also for general skin rejuvenation. There are numerous studies documenting the results of IPL treatment. Here we report our experience with the M-22 IPL device. The M-22 is the smallest device of its kind that delivers both IPL and Nd-Yag laser treatments. In our experience, the device is easy to handle, and based on unmeasured observation, it appears to be less painful for patients than the Vasculight (Lumenis, Yokneam, Israel), an earlier IPL model.

There are several reports of IPL technology being used for skin rejuvenation. ${ }^{8-10)}$ Most of the skin texture improvement reported is not long lasting. However, Weiss reported an 83\% improvement after a four year follow-up study. A combination of aminolevulinic acid (ALA) with IPL was reported to increase efficacy and results. ${ }^{11,12)}$ The high satisfaction rate in our study was probably due to our limiting the indications for the treatments to improving skin appearance by removing distinct pigmentary and vascular lesions, resulting in a general improvement of skin appearance. Taking into account our past experience with IPL technology, we excluded removal of skin wrinkles from the indications for this treatment, although some reports claim improvement in superficial wrinkles using this technology.

Weiss obtained an $82 \%$ improvement in teleangiectasia using IPL. ${ }^{13)}$ Other authors reported similar positive results for photorejuvenation with dominant vascular pattern. ${ }^{4,14,15)}$ The vascular lesions treated in this study were port wine stains and angiomas, which were treated using IPL technology. For telangiectasias, however, we preferred using the Yag laser mode, holding the head at a 45 degree angle; in these cases, patients reported a satisfaction rate of over $90 \%$.

Pigment improvement after IPL treatment was 


\section{ORIGINAL ARTICLES}

invariably reported. The target is the melanin within the melanosomes, with the melanosomes being directly affected by the heating of the melanin. The repigmentation that is observed after treatment, mainly after sun exposure, is associated with the residual melanocytes from the adnexal structures. ${ }^{16)}$ The superficial pigmented lesions have a better response to the treatment. Bjering ${ }^{17)}$ reported a $96 \%$ reduction in the pigmentary changes associated with lentigo solaris. Selecting patients with superficial pigmented lesions is important for obtaining a good clinical response with high satisfaction rates. Lentigines had the most effective response after a single IPL treatment in our study. Small lesions also had a better response to IPL treatment. ${ }^{18)}$ Epidermal melasma has a better response than mixed melasma. ${ }^{19)}$ We strongly recommend using the IPL for lesions such as café au lait, ephelides and epidermal melasma. Deeper lesions such as mixed melasmas and Baker nevi respond poorly to treatment.

\section{Conclusion}

There are many IPL devices on the market and their parameters vary widely from one device to another. The parameters of one device cannot be used safely for others, especially when manufactured by a different company. The first generation of IPL systems featured beam variations with the end of the pulse being close to the red/infrared spectrum; the newest devices employ a computer system and optimal pulse technology for improving results with less surrounding tissue damage.

In conclusion, the $\mathrm{M}-22$ is a small and compact device that can safely be used for different indications, with a short learning curve.

Disclosure: The authors have no financial interest in the device or the company. The M-22 device was supplied as a trade-in for an older, Vasculight version.

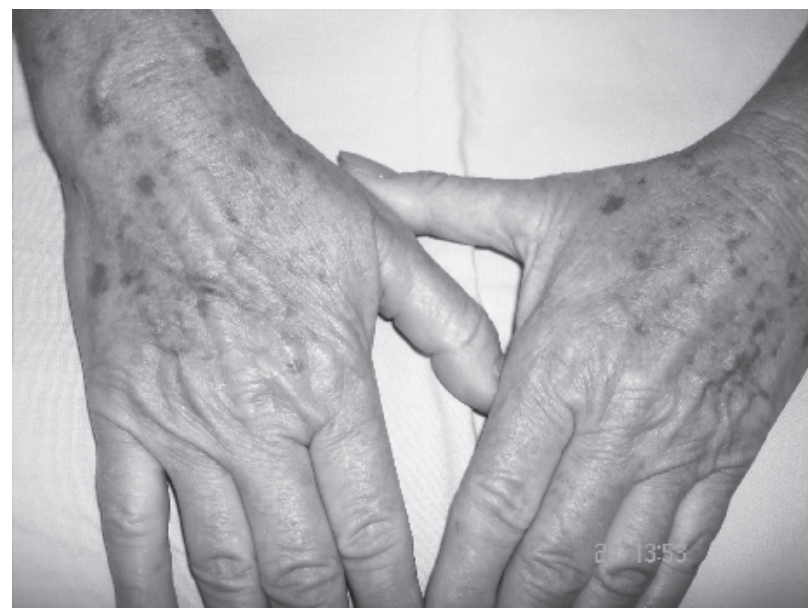

Figure 4: 64 year old woman with lentiginous hyperpigmentation.

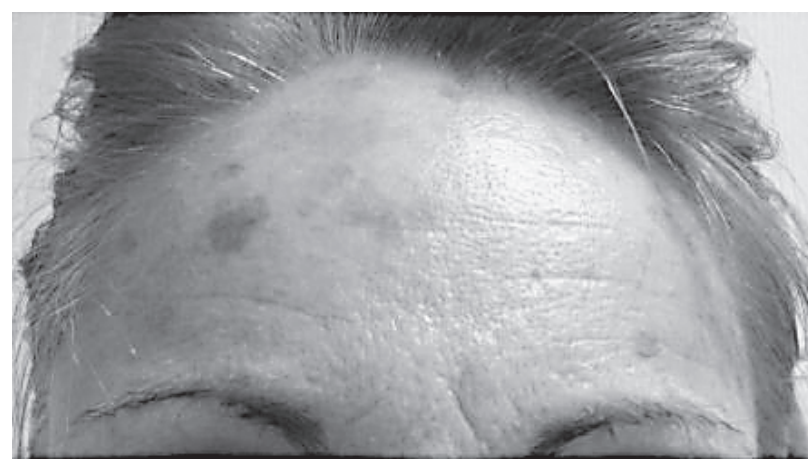

Figure 6: 51 year old woman with benign lentigo on the forehead

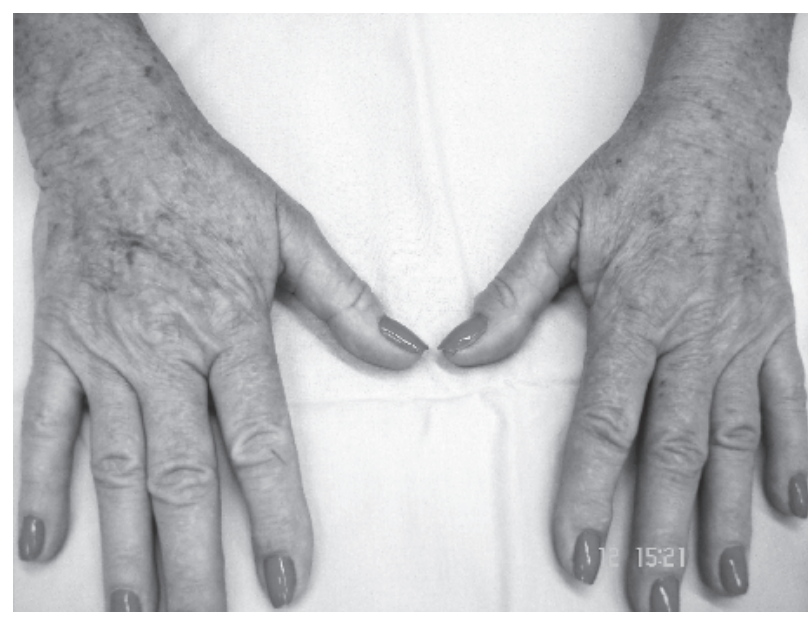

Figure 5: Same patient after 1 treatment

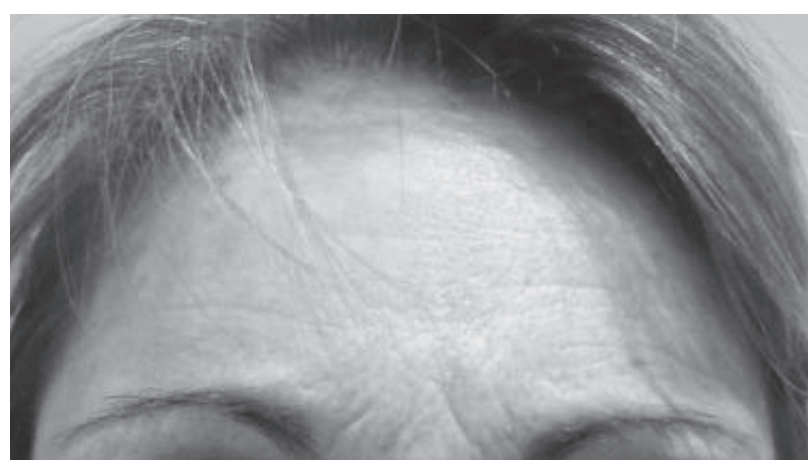

Figure 7: Same patient after 1 treatment 


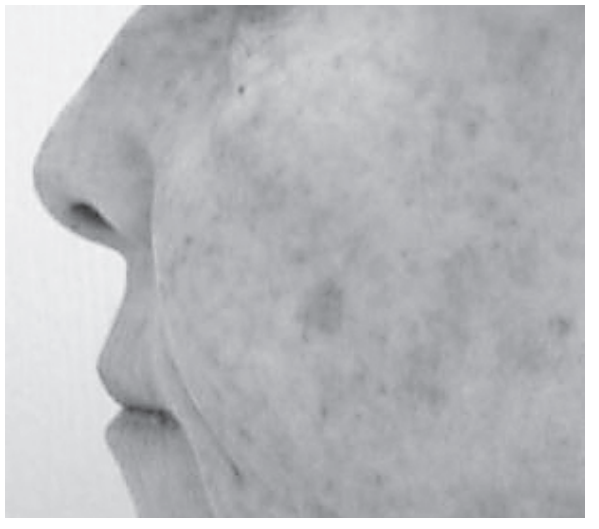

Figure 8: 58 year old woman with hyperpigmented sun-damage

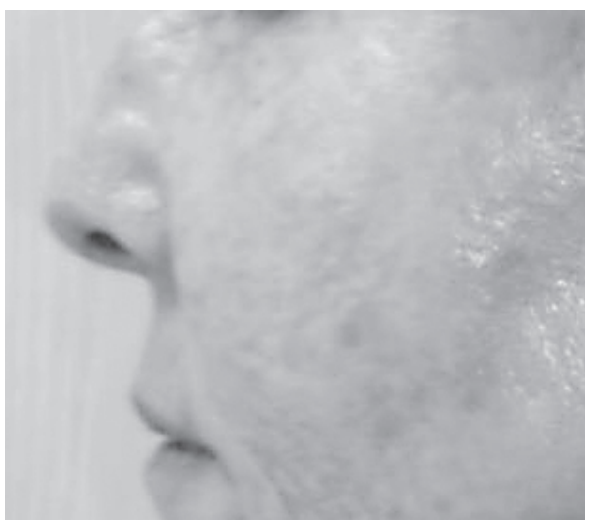

Figure 9: Same patient after 1 treatment

\section{References}

1: Anderson RR and Parrish JA (1981), The optics of human skin. Journal of Investigative Dermatology 77(1): 13-9.

2: Anderson RR and Parrish JA (1983) Selective photothermolysis: precise microsurgery by selective absorption of pulsed radiation. Science 220(4596): 524-7.

3: Liu H, Dang Y, Wang Z, Chai X and Ren Q (2008) Laser induced collagen remodeling: a comparative study in vivo on mouse model. Lasers in Surgery and Medicine 40(1): 13-9.

4: Negishi K, Wakamatsu S, Kushikata N, Tezuka Y, Kotani Y and Shiba K. (2002) Full-face photorejuvenation of photodamaged skin by intense pulsed light with integrated contact cooling: initial experiences in Asian patients. Lasers in Surgery and Medicine 30(4):298-305.

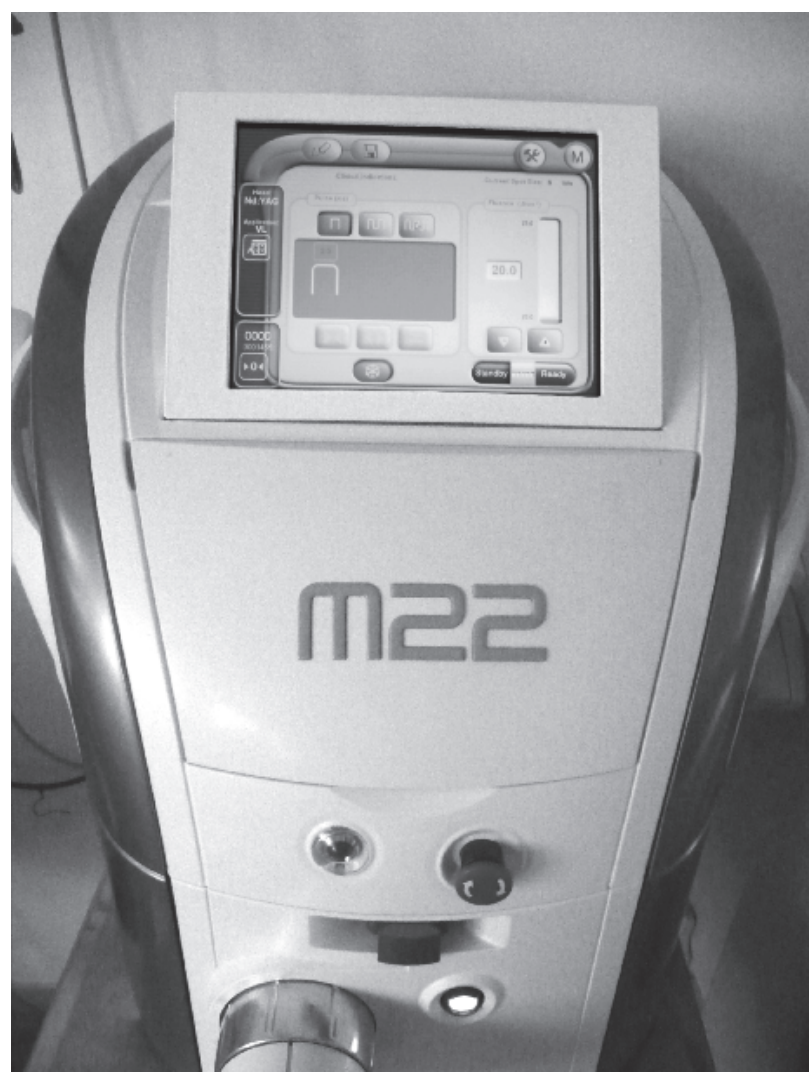

Figure 10: M-22 IPL Device (Lumenis Ltd., Yokneam, Israel)

5: Zelickson BK (2000) Effect of pulsed dye laser and intense pulsed light source on the dermal extracellular matrix remodeling. Lasers in Surgery and Medicine 12(Suppl): 17.

6: Goldberg DJ (2000) New collagen formation after dermal remodeling with an intense pulsed light source. Journal of Cutaneous Laser Therapy 2(2):59-61.

7: Yamashita T, Negishi K, Hariya T, Kunizawa N, Ikuta K, Yanai M and Wakamatsu S. (2006) Intense pulsed light therapy for superficial pigmented lesions evaluated by reflectance-mode confocal microscopy and optical coherence tomography. Journal of Investigative Dermatology 126(10):2281-6.

8: Nelson JS, Majaron B, and K.M. Kelly (2002) What is nonablative photorejuvenation of human skin? 
Seminars in Cutaneous Medicine and Surgery 21(4):238-50.

9: Fodor L, Peled IJ, Rissin Y, Ramon Y, Shoshani O, Eldor L, Gaiman A, Ullmann Y. (2004) Using intense pulsed light for cosmetic purposes: our experience. Plastic and Reconstructive Surgery 113(6):1789-95.

10: Bitter PH (2000) Noninvasive rejuvenation of photodamaged skin using serial, full-face intense pulsed light treatments. Dermatologic Surgery 26(9): 835-42; Discussion 843.

11: Gold MH and Goldman MP (2004) 5-aminolevulinic acid photodynamic therapy: where we have been and where we are going. Dermatologic Surgery 30(8):1077-83; Discussion 1083-4.

12: Taub AF (2004) Photodynamic therapy for the treatment of acne: a pilot study. Journal of Drugs in Dermatology 3(6 Suppl): S10-4.

13: Weiss RA, Weiss MA and Beasley KL (2002) Rejuvenation of photoaged skin: 5 years results with intense pulsed light of the face, neck, and chest. Dermatologic Surgery 28(12): 1115-9.

14: Negishi K, Tezuka Y, Kushikata N and Wakamatsu S (2001) Photorejuvenation for Asian skin by intense pulsed light. Dermatologic Surgery 27(7): 627-31; Discussion 632.

15: Goldman MP, Weiss RA, and Weiss MA (2005) Intense pulsed light as a nonablative approach to photoaging. Dermatologic Surgery 31(9 Pt 2):117987; Discussion 1187.

16: Margolis RJ, Dover JS, Polla LL, Watanabe S, Shea CR, Hruza GJ, Parrish JA and Anderson RR. (1989) Visible action spectrum for melanin-specific selective photothermolysis. Lasers in Surgery and Medicine 9(4): 389-97.

17: Bjerring P. and Christiansen K (2000) Intense pulsed light source for treatment of small melanocytic nevi and solar lentigines. Journal of Cutaneous Laser Therapy 2(4): 177-81.

18: Kawada A, Asai M, Kameyama H, Sangen Y, Aragane Y, Tezuka T and Iwakiri K. (2002) Clinical improvement of solar lentigines and ephelides with an intense pulsed light source. Dermatologic Surgery 28(6): 504-8.

19: Wang CC, Hui CY, Sue YM, Wong WR and Hong HS (2004) Intense pulsed light for the treatment of refractory melasma in Asian persons. Dermatologic Surgery 30(9): 1196-200. 